\title{
Global Earth's gravity field solution with GRACE orbit and range measurements using modified short arc approach
}

\author{
Qiujie Chen · Yunzhong Shen · Xingfu Zhang • \\ Houze Hsu • Wu Chen
}

Received: 14 March 2014 / Accepted: 29 October 2014 Published online: 15 November 2014

(C) Akadémiai Kiadó 2014

\begin{abstract}
Traditionally, the Earth's gravity field model is computed from GRACE orbit and range rate measurements, e.g., in a short arc approach where both the position and the velocity vectors are integrated from a force model. In this contribution, we use the GRACE orbit and range measurements to recover the Earth's gravity field model, thus we only need to integrate the position vectors. We use the range differences between two adjacent epochs to eliminate the range ambiguities. Using GRACE Level-1B RL02 data released by Jet Propulsion laboratory, the gravity field model TJGRACE02O complete to degree and order 90 is developed from 7 years of reduced dynamic orbits covering the period 2004-2010, and the gravity field model TJGRACE02K complete to degree and order 120 is computed from 1 month of kinematic orbits and K-band range data of January. Comparing the degree geoid errors of our new models with recent gravity field models such as the CHAMP-only models EIGEN-CHAMP05S, AIUB-CHAMP03S, ULUX-CHAMP2013S and the GRACE-only models GGM05S, Tongji-GRACE01 as well as a monthly model from the ITG-GRACE2010
\end{abstract}

Q. Chen · Y. Shen $(\bowtie)$

College of Surveying and Geo-informatics, Tongji University, Shanghai 200092,

Peolple's Republic of China

e-mail: yzshen@tongji.edu.cn

Q. Chen · W. Chen

Department of Land Surveying and Geo-Informatics, Hong Kong Polytechnic University,

Hong Kong, Peolple's Republic of China

Q. Chen · Y. Shen

Center for Spatial Information Science and Sustainable Development,

Tongji University, Shanghai 200092, Peolple's Republic of China

X. Zhang

Department of Surveying and Mapping, Guangdong University of Technology,

Guangzhou 510006, Peolple's Republic of China

H. Hsu

State Key Laboratory of Geodesy and Earth's Dynamics, Institute of Geodesy and Geophysics, CAS, Wuhan 430077, Peolple's Republic of China 
time series, and validating these models with GPS-leveling data sets in the USA, we can conclude that the TJGRACE02O model is more accurate than all the CHAMP-only models and TJGRACE02K is comparable in quality to the corresponding GRACE monthly model from ITG-GRACE2010.

Keywords Modified short arc approach - Satellite orbit - Satellite gravimetry · GRACE

\section{Introduction}

Recovering Earth's gravity field models by using the data from Challenging Mini-satellite Payload (CHAMP) (Reigber et al. 2002), Gravity Recovery and Climate Experiment (GRACE) (Tapley et al. 2004) and Gravity Field and steady State Ocean Circulation Explorer (GOCE) (Drinkwater et al. 2006) has been a hot research area for satellite geodesy in recent years. Especially the GRACE mission, launched on March 17, 2002, has been successfully applied to reflect the static Earth's gravity field up to degree and order 180, because the GRACE mission is very sensitive to the Earth's gravity field signals at medium and long wave lengths. By using the observations of CHAMP, GRACE and GOCE, more than 60 static Earth's gravity field models have already been developed, which are available on the International Center for Global Earth Models (ICGEM) website (http://icgem. gfz-potsdam.de/ICGEM/), and can be assorted as follows: the CHAMP-only models including EIGEN-CHAMP05S (Flechtner et al. 2010) and AIUB-CHAMP03S (Prange et al. 2011), the GRACE-only models such as GGM05S (Tapley et al. 2013), AIUB-GRACE03S (Jäggi et al. 2010), ITG-GRACE2010S (Mayer-Gürr et al. 2010) and Tongji-GRACE01 (Shen et al. 2013), the GOCE-only model ITG-GOCE02 (Schall et al. 2014) and the combined GRACE/GOCE models including DGM-1S (Farahani et al. 2013) and EIGEN6C2 (Förste et al. 2013). The main methodologies for developing these models are the dynamic approach, the celestial mechanics approach (Beutler et al. 2010a, b), the short arc approach (Mayer-Gürr et al. 2010) and the acceleration approach (Farahani et al. 2013).

The short arc approach was recently modified by Shen et al. (2013). In this modified short arc approach the kinematic orbits or reduced dynamic orbits are regarded as pseudo observations, and the observation equation is linearized with respect to the pseudo observations as described in Chen et al. (2013). Then we compute the gravity field model and arc-specific parameters, and estimate the observation errors with the linearized observation equation using a least squares adjustment. In this case, the gradient corrections applied by Mayer-Gürr et al. (2010) are no longer needed. The modified short arc approach has been applied by Chen et al. (2014) to develop the Tongji-GRACE01 gravity field model from GRACE K-band range rate data, reduced dynamic orbits, attitude and acceleration measurements. However, to establish the observation equations for range rate and orbit measurements, both the position and velocity vectors of the GRACE satellites are needed to be integrated using a force model. If the range rate measurements are replaced by differences between adjacent range measurements, the velocity integration is no longer needed. For this reason, we investigate the gravity field solution from orbit and range measurements by using the further modified short arc approach, as it is described in Sect. 2 and is implemented in the Satellite Gravimetry Analysis Software (SAGAS) by Tongji University. This article is outlined as follows. In Sect. 2, we will describe the theoretical model of our modified short arc approach. In Sect. 3, details of the processing of GRACE data are sketched. Section 4 assesses the determined gravity models by comparison to alternative models and validation with GPS-leveling data sets. Section 5 is devoted to the conclusions. 


\section{Observation equation and data processing method}

\subsection{Position and range observation equations in the modified short arc approach}

The position vector of the kinematic orbit or reduced dynamic orbit is regarded as pseudo observations in the modified short arc approach. However, unlike the traditional short arc approach which carries out a gradient correction to the kinematic or reduced dynamic orbit (Mayer-Gürr 2006), the position observation equation reads (Shen et al. 2013),

$$
\begin{aligned}
\boldsymbol{r}(\tau)+\boldsymbol{v}_{r}(\tau)= & \left(\boldsymbol{r}_{0}+\boldsymbol{v}_{r_{0}}\right)(1-\tau)+\tau\left(\boldsymbol{r}_{N}+\boldsymbol{v}_{r_{N}}\right) \\
& -T^{2} \int_{0}^{1} K\left(\tau, \tau^{\prime}\right) \boldsymbol{a}\left(\boldsymbol{r}\left(\tau^{\prime}\right)+\boldsymbol{v}_{r}\left(\tau^{\prime}\right), \boldsymbol{u}, \boldsymbol{p}\right) d \tau^{\prime}
\end{aligned}
$$

with the same integral kernel function used by Mayer-Gürr (Mayer-Gürr 2006),

$$
K\left(\tau, \tau^{\prime}\right)= \begin{cases}\tau\left(1-\tau^{\prime}\right), & \tau \leq \tau^{\prime} \\ \tau^{\prime}(1-\tau), & \tau \geq \tau^{\prime}\end{cases}
$$

where, $\mathbf{r}(\tau)$ and $\mathbf{v}_{r}(\tau)$ stand for the position vector of the kinematic or reduced dynamic orbit and the corresponding correction vector at normalized time $\tau ; \mathbf{r}_{0}$ and $\mathbf{r}_{N}$ are the position vectors at the two boundaries of the integral arc and $\mathbf{v}_{r_{0}}$ and $\mathbf{v}_{r_{N}}$ are the corresponding correction vectors; $\mathbf{a}\left(\mathbf{r}(\tau)+\mathbf{v}_{r}(\tau), \mathbf{u}, \mathbf{p}\right)$ stands for the force acting on the satellite; $\mathbf{u}$ and $\mathbf{p}$ are the geopotential coefficients and the accelerometer bias parameters, respectively. The $\mathrm{K}$-band range measurement can be expressed as,

$$
\rho(\tau)+\boldsymbol{v}_{\rho}(\tau)=\delta \rho+\boldsymbol{e}_{A B}^{T}(\tau)\left(\boldsymbol{r}_{B}(\tau)-\boldsymbol{r}_{A}(\tau)\right)
$$

where $\rho(\tau)$ stands for the K-band range measurements between GRACE $A$ and $B, \mathbf{v}_{\rho}(\tau)$ is the corresponding correction term, $\delta \rho$ is the range ambiguity, $\mathbf{e}_{A B}(\tau)$ is the unit vector of the line of sight from GRACE $A$ to $B$, and $\mathbf{r}_{A}(\tau)$ and $\mathbf{r}_{B}(\tau)$ are the position vectors of the twin GRACE satellites. By substituting Eq. (1) into Eq. (3), we can establish the observation equation for the K-band range measurement. Then by using the position and range observation equations, we can solve for the geopotential coefficients $\mathbf{u}$ and the accelerometer bias $\mathbf{p}$ as well as the correction vectors $\mathbf{v}_{r}(\tau)$ and $\mathbf{v}_{\rho}(\tau)$ by a least squares adjustment. By using the difference between two ranges at epochs with the same ambiguity, we can eliminate the range ambiguity parameter.

The range and range rate measurements, e.g. $\rho(\tau)$ and $\dot{\rho}(\tau)$, are alternatively used to form the observation equation. If range rate measurements are used as in Shen et al. (2013), additionally the velocity integration has to be implemented,

$$
\dot{\boldsymbol{r}}(\tau)=\left(\boldsymbol{r}_{N}+\boldsymbol{v}_{r_{N}}-\boldsymbol{r}_{0}-\boldsymbol{v}_{r_{0}}\right) / T+T \int_{0}^{1} \frac{\partial K\left(\tau, \tau^{\prime}\right)}{\partial \tau} \boldsymbol{a}\left(\boldsymbol{r}\left(\tau^{\prime}\right)+\boldsymbol{v}_{r}\left(\tau^{\prime}\right), \boldsymbol{u}, \boldsymbol{p}\right) d \tau^{\prime}
$$

where, $\frac{\partial K\left(\tau, \tau^{\prime}\right)}{\partial \tau}$ is the partial derivative of Eq. (2). Using the range measurements to substitute the range rate measurements for computing the gravity field model, we can avoid the velocity integration and therefore make the software more efficient.

\subsection{Data sets and background force models}

The official GRACE level-1B observations released by Jet Propulsion Laboratory (JPL), containing reduced dynamic orbits ( $5 \mathrm{~s}$ sample rate), K-band range (5s sample rate), attitudes (5s sample rate) and non-conservative accelerations (1s sample rate), have been used to 
compute our gravity field models. Additionally, because the reduced dynamic orbits contain a priori information on the gravity field, one month of kinematic orbits with 30s sample rate from the GNSS Research Centre of Wuhan University (Zhao 2004) also is adopted for comparisons. The absolute accuracy of the kinematic orbit is at the level of $4 \mathrm{~cm}$ (Zhao et al. 2011). Note that the accelerometer and kinematic orbit data should be downsampled to 5 $\mathrm{s}$ beforehand in order to keep consistent with the other types of GRACE measurements and the step size of the numerical integration $(5 \mathrm{~s})$. The relative weighting of the observations, i.e. the orbits and range differences, is determined by using their priori accuracies, namely $3 \mathrm{~cm}$ and $1.0 \mu \mathrm{m}$ (Beutler et al. 2010b).

In the processing procedures of gravity field recovery, the background forces are computed with the existing force models, including general relativistic perturbations, solid earth tides, solid pole tides, ocean tides, ocean pole tides, atmosphere and ocean mass variability, N-Body perturbations and the non-conservative force. In this paper, general relativistic perturbations, solid earth tides and solid pole tides are computed according to the IERS2010 conventions. Ocean tides are calculated by EOT11a (Savcenko and Bosch 2012; Rieser et al. 2012) complete to degree and order 80, in which 18 main waves are included and the secondary waves are interpolated with admittance theory (Rieser et al. 2012). Applying the Desai model (Desai 2002) up to degree and order 30 , the effect of ocean pole tides is removed. The atmosphere and oceanic de-aliasing Level-1B Release 05 product (AOD1B RL05) complete to degree and order 100 is employed to correct the impact of the atmosphere and ocean mass variability (Flechtner and Dobslaw 2013). N-Body perturbations caused by the Sun, Moon and other planets are directly computed according to IERS2010, of which both the direct effect and the indirect $J_{2}$ effect on the spacecraft are considered according to the GRACE Level-2 standard document (Dahle et al. 2012) from GeoForschungsZentrum (GFZ). The non-conservative force is directly measured by the GRACE onboard accelerometers.

Like the traditional short arc approach, the modified short arc approach does not depend on a priori gravity field. Thus no a priori gravity field is needed to recover the gravity field model. The high degree coefficients not recovered are taken over in a priori force model from the excellent EIGEN6C2 model which was determined from the combined data of GRACE, GOCE, laser geodynamics satellite (Lageos), terrestrial and air borne gravity data and altimetry data (Förste et al. 2013) in order to avoid spectral omission.

\subsection{Determination of arc-specific parameters and arc length}

The main arc-specific parameters are the K-band range ambiguities (Liu 2008) and the bias and scale parameters of the acceleration measurements. Since the ambiguity will change at some epochs, we use the range differences of adjacent epochs to eliminate the range ambiguity parameters for convenience in data processing.

The non-conservative forces measured by the accelerometers are biased and affected by drifts (Beutler et al. 2010b), that have to be accounted for by bias and scale parameters. However, the drift is less significant and more correlated with secular geopotential coefficients than the bias (Helleputte et al. 2009). Therefore, GFZ and Bonn University only estimate the bias parameters in the computation of the excellent EIGEN6C2 and ITG-GRACE2010S models, and we will also only estimate bias parameters in our solution. The arc length also has a significant impact on the final gravity field solution and varies considerably in different approaches, GFZ, for example, computed EIGEN6C2 using the traditional dynamic approach using one day arcs, while Bonn University derived ITG-GRACE2010S using the traditional short arc approach with one hour arcs. In order to determine the most appropriate arc length for our gravity field solution, where K-band rang measurement is not used, we have computed 


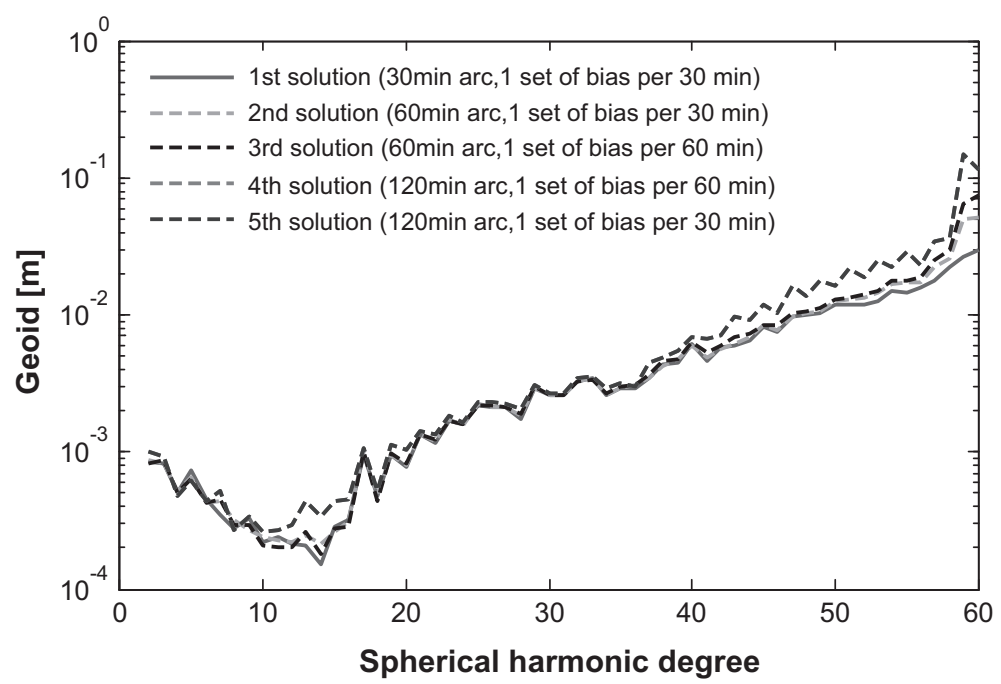

Fig. 1 Difference degree variances of five solutions with different arc-lengths, relative to the EIGEN6C2. No K-band data was used

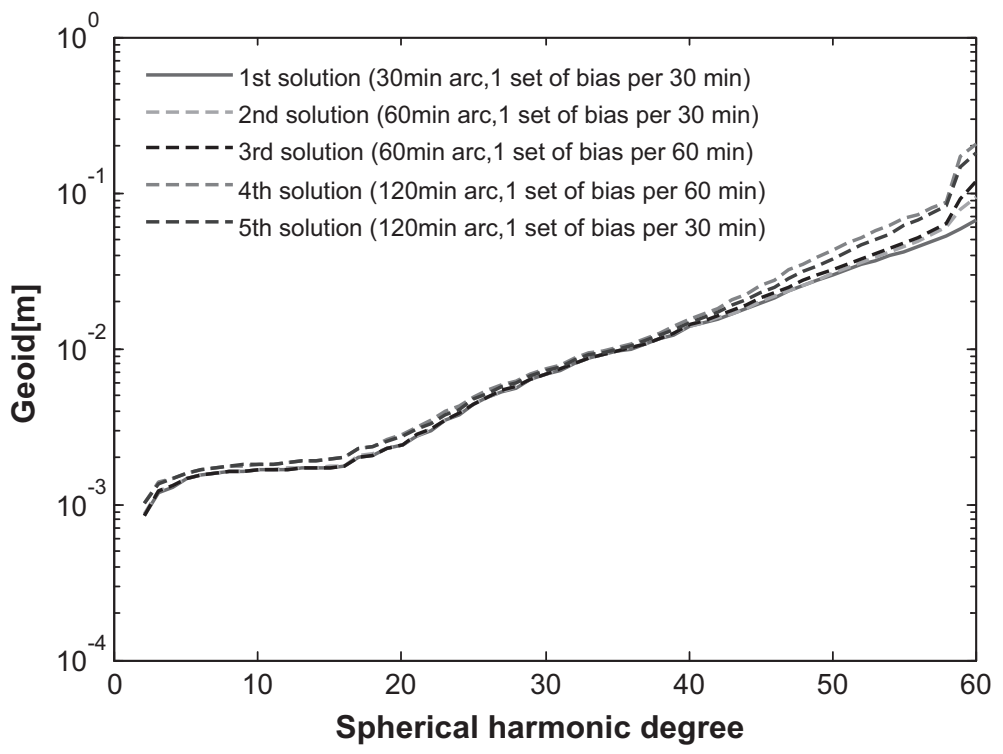

Fig. 2 Cumulative geoid errors relative to EIGEN6C2

five different solutions using the GRACE orbits, attitudes and accelerations of January 2008. The results are shown in Fig. 1 and 2 in terms of difference degree variances and cumulative geoid error relative to EIGEN6C2. In the first solution, the arc length is taken as $30 \mathrm{~min}$ and one set of biases for the three axes (Along-track, Cross-track, Radial) for each GRACE satellite is estimated per arc. The other four solutions with different arc lengths are also presented in Fig. 1 and 2. It is obvious that the first solution performs best. Thus we take over this parameterization to derive the static gravity field model TJGRACE02O from GRACE orbits, 


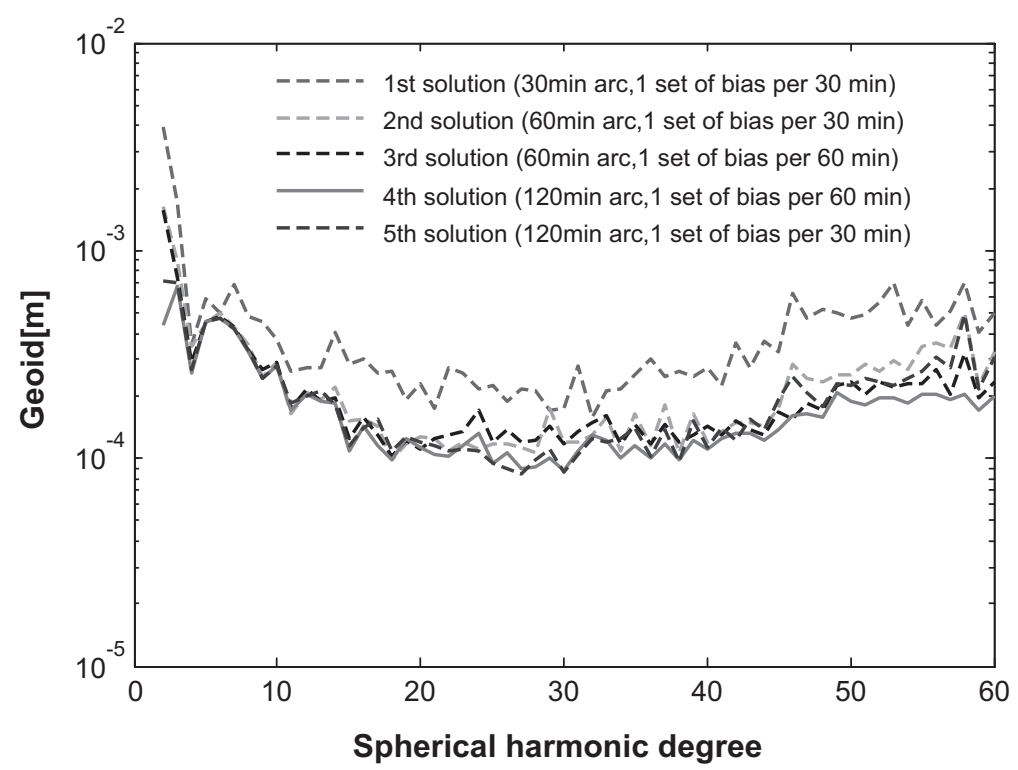

Fig. 3 Difference degree variances of five solutions with different arc lengths, relative to the EIGEN6C2. This time K-band data was used

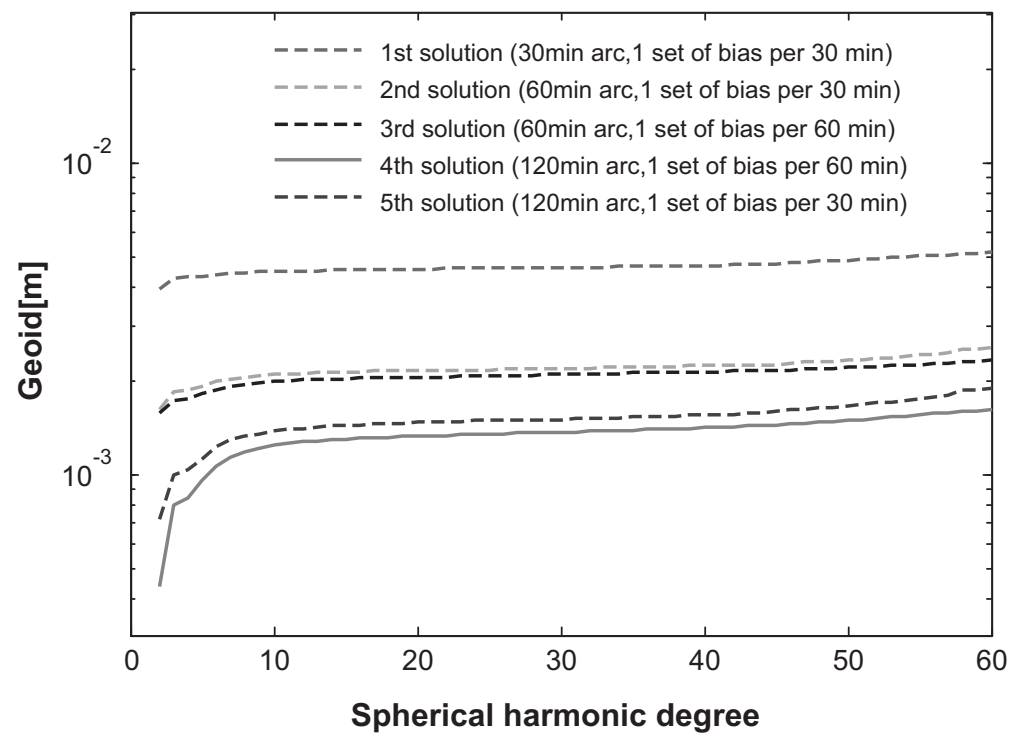

Fig. 4 Cumulative geoid errors relative to EIGEN6C2

attitude and acceleration data. We performed the same experiments to compute gravity field solutions where K-band rang measurements are included, the results are represented in Fig. 3 and 4 . Arc lengths beyond $2 \mathrm{~h}$ are not considered due to the time consuming computation. We find that the solution with 120 min arc-length and one set of bias parameters every 60 min is the best solution, and therefore this choice is used to compute the static gravity field model TJGRACE02K using GRACE data including K-band rang measurements. 


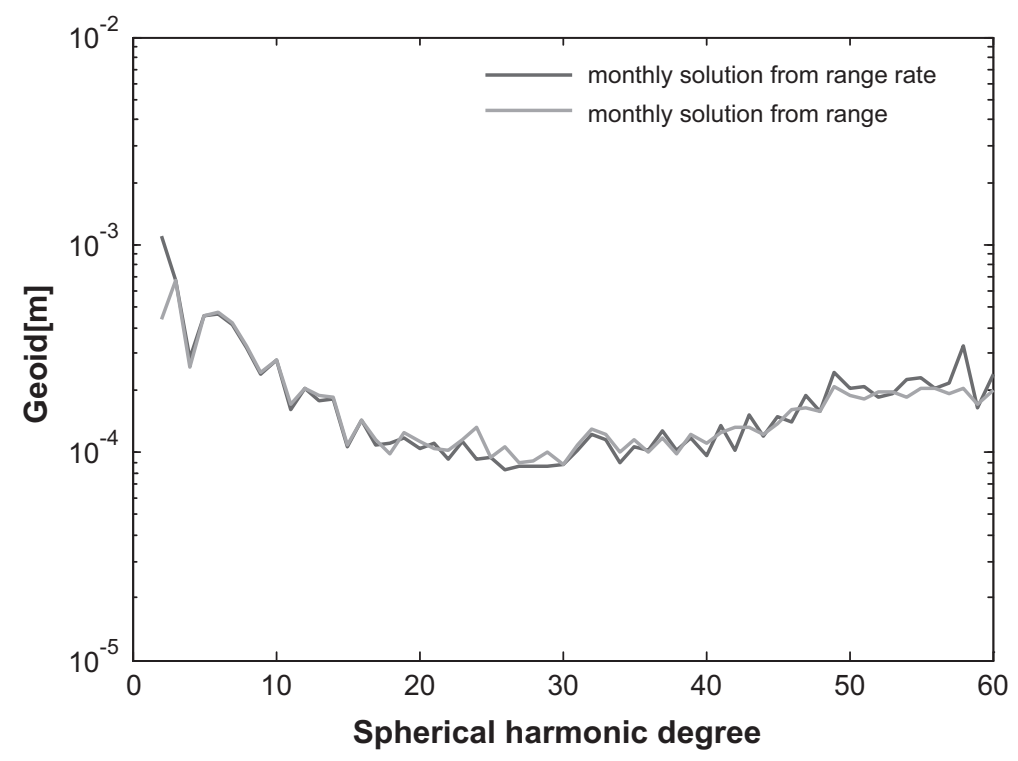

Fig. 5 Difference degree variances of monthly solutions of January 2008 from range rate or range data, relative to EIGEN6C2

\subsection{Comparisons of solutions using K-band range or K-band range rate observations}

The recent GRACE monthly gravity solutions Tongji-GRACE01 are also computed by using the modified short arc approach but with GRACE K-band range rate observations, therefore both the position and velocity of the GRACE A and GRACE B satellites have to be integrated. If we use the K-band range observations to compute the gravity field model, the integration of velocity is not necessary, making the program more efficient. Fig. 5 shows the difference degree variances of monthly solutions complete to degree and order 60 from range rate or range observations. The quality of the two solutions is almost the same.

\subsection{Comparisons of solutions from reduced dynamic or kinematic orbits}

As mentioned above, the reduced dynamic orbits contain a priori information of the gravity field. In an attempt to look into the impact of the reduced dynamic orbits on the GPS-only or K-band solution, we have computed two GPS-only models and two K-band models by using either 1 month of reduced dynamic orbits or one month of kinematic orbits. The results are represented in Fig. 6. The GPS-only solution using reduced dynamic orbit shows a significantly better performance compared to that using the kinematic orbits. This is due to a priori gravity field information contained in the dynamic orbits. In case of K-band range data, the results using reduced dynamic or with kinematic orbits are consistent, indicating that the gravity field is determined by the K-band measurement, while the impact of the reduced dynamic orbits is negligible.

\section{Static gravity field models TJGRACE02SO and TJGRACE02SK}

According to the modified short arc approach and the processing procedure described above, the static gravity field model TJGRACE02O complete to degree and order 90 is successfully 


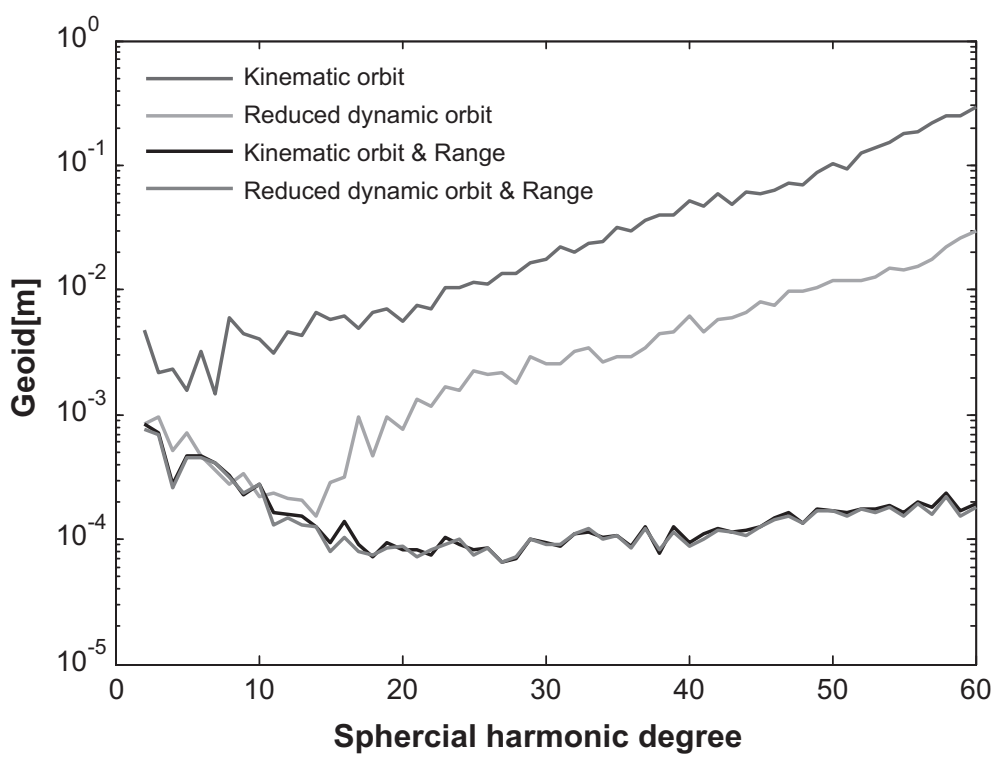

Fig. 6 Comparisons between GPS-only and K-band solution of Jan. 2008 derived using either reduced dynamic or kinematic orbits
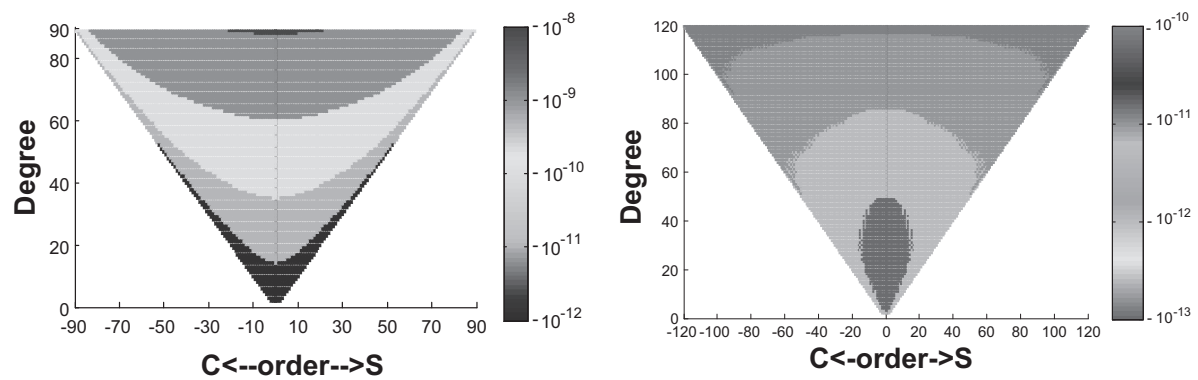

Fig. 7 The formal errors of the coefficients of TJGRACE02O (left figure) and TJGRACE02K (right figure)

computed by using the GRACE observations including reduced dynamic orbits, attitudes and accelerations from 2004 to 2010, in total almost 7 years of data. The data of January 2004 is excluded due to data gaps. The gravity field model TJGRACE02K complete to degree and order 120 is derived from GRACE data including kinematic orbits, K-band range, attitude and acceleration measurements in January 2008. We compare our model to, amongst others, Tongji-GRACE01 that was determined using GRACE data from January 2003 to October 2011 , i.e., from a time span much longer.

The formal errors of the coefficients of TJGRACE02O and TJGRACE02K models are represented in Fig. 7. We conclude that the formal errors of TJGRACE02O coefficients at degrees up to 40 are less than $10^{-10}$, while those of TJGRACE02K coefficients up to degree 60 are less than $10^{-11}$, indicating that the K-band rang data has significantly contributed to the gravity field solution. For further assessment of TJGRACE02O and TJGRACE02K, we compare the difference degree variances relative to the superior model EIGEN6C2 and cumulative geoid errors of these two models to state of the art gravity field models. TJGRACE02O is 


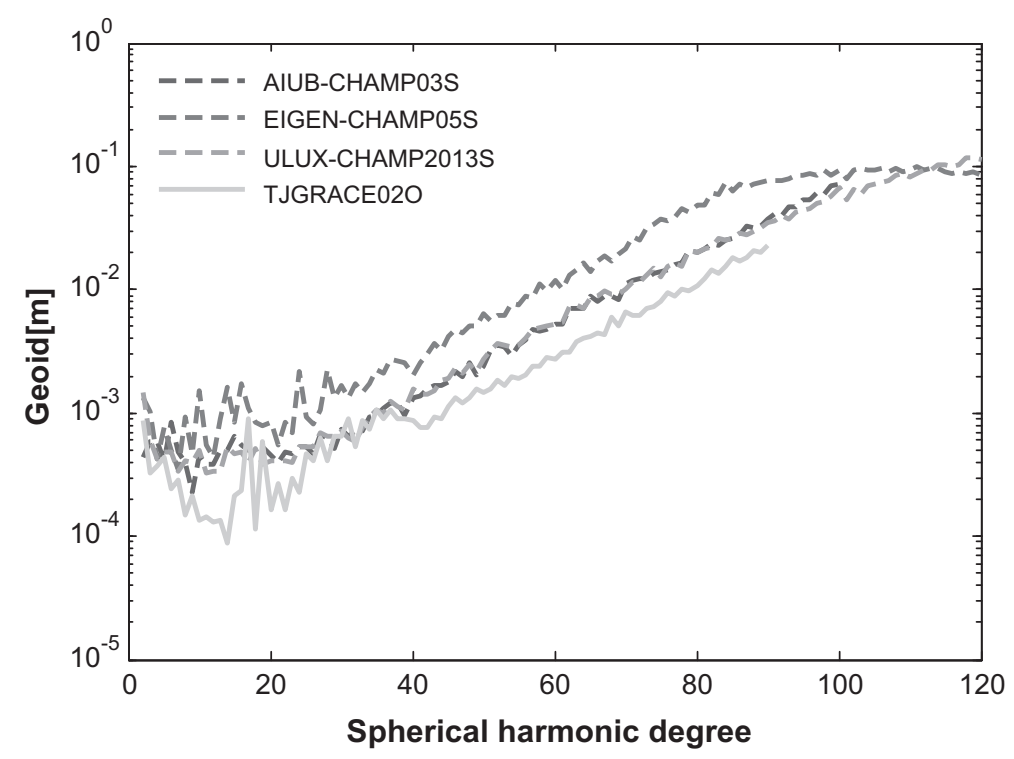

Fig. 8 Difference degree variances of GPS-only solutions relative to EIGEN6C2

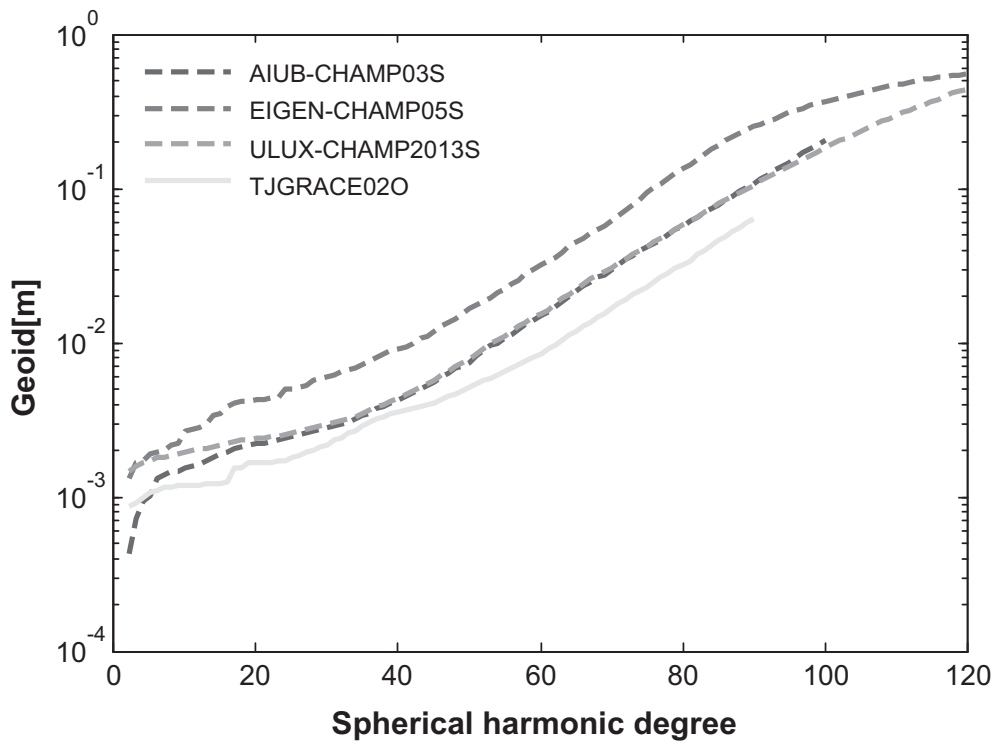

Fig. 9 Cumulative geoid errors of GPS-only solutions relative to EIGEN6C2

compared to the CHAMP-only models EIGEN-CHAMP5S, AIUB-CHAMP03S and ULUXCHAMP2013S. TJGRACE02K is compared to the long term K-band models GGM05S, Tongji-GRACE01 and to the monthly ITG-GRACE2010 model of the corresponding month. The results are shown in Fig. 8, 9, 10 and 11. We can see from Fig. 8 and 9 that TJGRACE02O performs better than all the GPS-only models AIUB-CHAMP03S, EIGEN-CHAMP05S and ULUX-CHAMP2013S up to degree and order 90. But the good performance of our GPS-only 


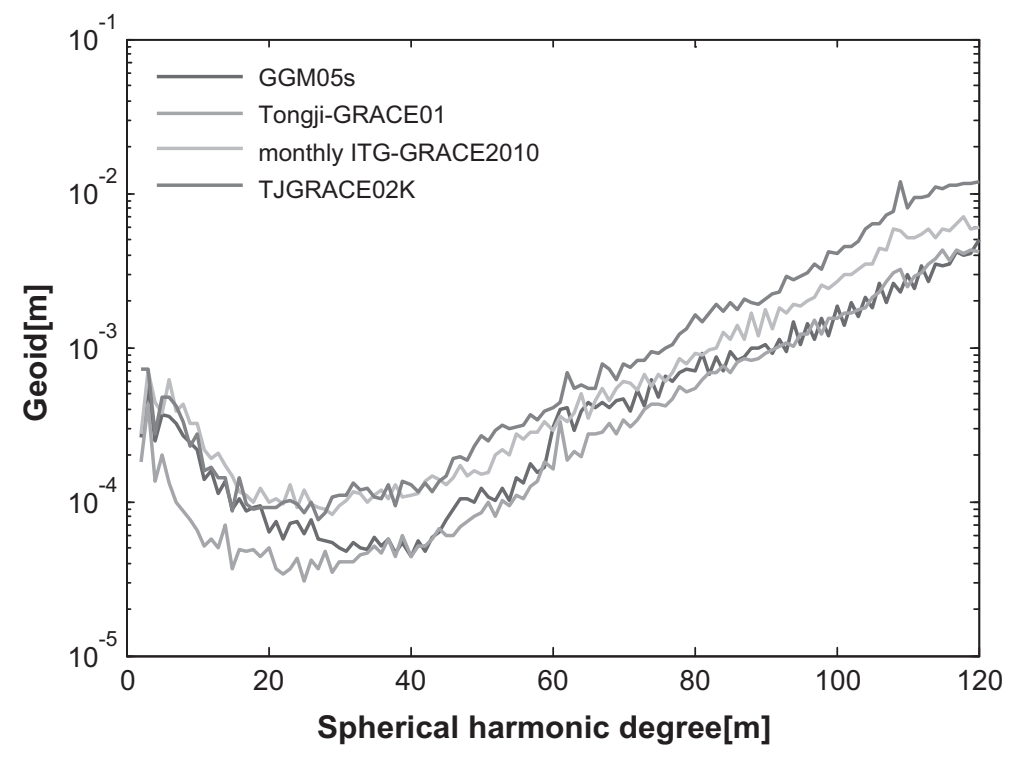

Fig. 10 Difference degree variances of K-band solutions relative to EIGEN6C2

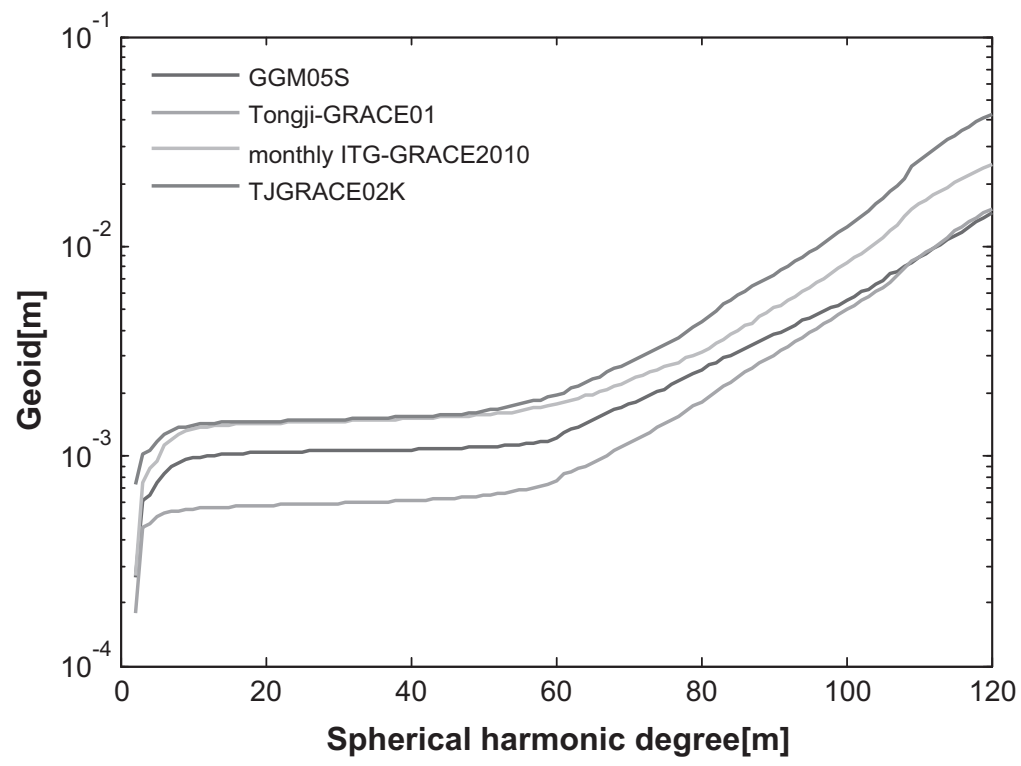

Fig. 11 Cumulative geoid errors of K-band solutions relative to EIGEN6C2

model is partly contributed by a priori information of gravity field because we use the reduced dynamic orbits. From Fig. 10 and 11, we conclude that the accuracy of TJGRACE02K from one month of K-band range data is comparable to that of the monthly ITG-GRACE2010 model. Understandably the quality is inferior to that of static fields GGM05S and TongjiGRACE01 based on much longer data spans. Tables 1 and 2 list the cumulative geoid errors 
Table 1 Cumulative geoid errors for GPS-only solutions (cm)

\begin{tabular}{lllll}
\hline Degree & AIUB-CHAMP & $\begin{array}{l}\text { EIGEN-CHAMP } \\
\text { 05S }\end{array}$ & $\begin{array}{l}\text { ULUX-CHAMP } \\
2013 \mathrm{~S}\end{array}$ & $\begin{array}{l}\text { TJGRACE } \\
02 \mathrm{O}\end{array}$ \\
\hline 30 & $03 \mathrm{~S}$ & 0.6 & 0.3 & 0.2 \\
60 & 0.3 & 3.2 & 1.5 & 0.8 \\
90 & 1.5 & 25.1 & 10.5 & 6.4 \\
120 & 10.7 & 55.1 & 44.3 & $*$ \\
\hline
\end{tabular}

* Denotes unavailable for test

Table 2 Cumulative geoid errors for K-band solutions $(\mathrm{cm})$

\begin{tabular}{lllll}
\hline Degree & GGM05S & Tongji-GRACE01 & Monthly ITG-GRACE2010 & $\begin{array}{l}\text { TJGRACE } \\
\text { 02K }\end{array}$ \\
\hline 30 & 0.1 & 0.1 & 0.1 & 0.1 \\
60 & 0.1 & 0.1 & 0.2 & 0.2 \\
90 & 0.4 & 0.3 & 0.5 & 0.7 \\
120 & 1.5 & 1.5 & 2.4 & 4.2 \\
\hline
\end{tabular}

Table 3 Root mean square (RMS) of differences geoid height $(\mathrm{cm})$ between GPS-leveling and GPS-only models truncated at different degrees

\begin{tabular}{lllll}
\hline Degree & AIUB-CHAMP & $\begin{array}{l}\text { EIGEN-CHAMP } \\
\text { 05S }\end{array}$ & $\begin{array}{l}\text { ULUX-CHAMP } \\
\text { 2013S }\end{array}$ & TJGRACE 02O \\
\hline 30 & 21.7 & 21.6 & 21.7 & 21.7 \\
60 & 21.7 & 21.5 & 21.9 & 21.8 \\
90 & 25.6 & 32.7 & 29.0 & 22.7 \\
120 & $*$ & 45.3 & 53.6 & $*$ \\
\hline
\end{tabular}

* Denotes unavailable for test

of the GPS-only models and K-band models truncated at degrees 30, 60, 90, 120. The good performance of TJGRACE02O and TJGRACE02K is confirmed.

\section{Validations with external data}

To further demonstrate the accuracy of TJGRACE02O and TJGRACE02K, the 8006 GPSleveling points in the United State, which can be downloaded freely through the website (http://www.ngs.noaa.gov/GEOID/GEOID12), are taken to compute the RMS between the GPS-leveling and the model geoid heights of the GPS-only solutions and K-band solutions truncated at different degrees (e.g. 30, 60, 90, 120 degrees, degree 120 is unavailable for AIUB-CHAMP03S and TJGRACE02O). The results are shown in Tables 3 and 4. To avoid omission errors the coefficients of high degree and order are replaced by EIGEN6C2 coefficients complete to degree and order 1949. From Tables 3 and 4 we conclude that all GPS-only models and K-band models show almost the same accuracy up to degree 60 . When truncated at degree 90, the GPS-only models are significantly different and TJGRACE02O performs better than the CHAMP only models. But, as we mention above, the good performance of 
Table 4 Root mean square (RMS) of differences geoid height $(\mathrm{cm})$ between GPS-leveling and K-band models geoid heights truncated at different degrees

\begin{tabular}{lllll}
\hline Degree & GGM05S & Tongji-GRACE01 & Monthly ITG-GRACE2010 & TJGRACE 02K \\
\hline 30 & 21.7 & 21.7 & 21.7 & 21.7 \\
60 & 21.7 & 21.7 & 21.7 & 21.7 \\
90 & 21.9 & 21.6 & 21.8 & 21.8 \\
120 & 22.1 & 21.7 & 22.2 & 23.0 \\
\hline
\end{tabular}

our GPS-only model, to some extent, is affected by a priori information of gravity field contained in the reduced dynamic orbits. Conversely, less differences can be found comparing the K-band models truncated at degree 90 or 120.

\section{Conclusions}

In this paper the computation of the static gravity field models TJGRACE02O, complete to degree and order 90 from 7 years of orbit data, and TJGRACE02K, complete to degree and order 120 from one month of orbit and K-band range data, is described. The method applied is the modified short arc approach. The modified short arc approach uses only range measurement and therefore the velocity integration can be avoided. The comparisons of difference degree variances and the validation with GPS-leveling data demonstrate that TJGRACE02O performs better than CHAMP-only models and TJGRACE02K performs nearly as good as the corresponding monthly model from ITG-GRACE2010. These results demonstrate that the GRACE K-band range data is the most significant contribution to gravity solution (compared to GRACE orbit data). In the future, the GRACE range observations from 2003 to 2013 will be used to recover the static gravity field model complete to degree and order 180, which is expected to further improve the accuracy of our model. Also a time series of monthly solution complete to degree and order 60 spanning from 2003 to 2013 will be determined by using range observations.

Acknowledgments This paper was mainly sponsored by National Natural Science Foundation of China (41474017) and National Key Basic Research Program of China (973 Program; 2012CB957703), it is also partly supported by National Natural Science Foundation of China (41274035, 41104002). It is also sponsored by State Key Laboratory of Geodesy and Earth's Dynamics (SKLGED2013-3-2-Z, SKLGED2014-1-3-E). Thanks for JPL providing us the GRACE level-1B observations and professor Qile Zhao from the GNSS Research Centre of Wuhan University for providing us one month of kinematic orbit data. We also are grateful to two anonymous reviewers' comments and one professor from English department of The Hong Kong Polytechnic University for improvement of our original manuscript.

\section{References}

Beutler G, Jäggi A, Mervart L, Meyer U (2010a) The celestial mechanics approach: theoretical foundations. J Geod 84(10):605-624

Beutler G, Jäggi A, Mervart L, Meyer U (2010b) The celestial mechanics approach: application to data of the GRACE mission. J Geod 84(11):661-681

Chen Q, Shen Y, Zhang X (2013) Linearization method of recovering Earth's gravity field with respect to gravity satellite's kinematic orbits. Chin J Geophys 56(7):2238-2244

Chen Q, Shen Y, Hsu H, Chen W (2014) Tongji-GRACE01-A GRACE-only Static Gravity Field Model Recovered From GRACE Level-1B Observations using Modified Short Arc Approach. Advances in Space Research. (paper in preparation) 
Dahle C, Flechtner F, Gruber C, König D, König R, Michalak G, Neumayer KH (2012) GFZ GRACE Level-2 Processing Standards Document for Level-2 Product Release 0005. Scientific Technical Report-Data, 12

Desai SD (2002) Observing the pole tide with satellite altimetry. J Geophys Res 107(C11):7

Drinkwater MR, Haagmans R, Muzi D, Popescu A, Floberghagen R, Kern M, Fehringer M (2006) The GOCE gravity mission: ESA's first core explorer. ESASP-627. ESA Publication Division, Noordwijk

Farahani HH, Ditmar P, Klees R, Liu X, Zhao Q, Guo J (2013) The static gravity field model DGM-1S from GRACE and GOCE data: computation, validation and an analysis of GOCE mission's added value. J Geod 87(9):843-867

Flechtner F, Dahle C, Neumayer KH, König R, Förste C (2010) The release 04 CHAMP and GRACE EIGEN gravity field models. Syst Earth via Geod Geophys Space Tech, 41-58

Flechtner F, Dobslaw H (2013) AOD1B Product Description Document for Product Release. 05. GFZ German Research Centre for Geosciences

Förste C, Bruinsma S, Flechtner F, Marty JC, Dahle C, Abrykosov O, Lemoine JM, Neumayer H, Barthelmes F, Biancale R, König R (2013) EIGEN-6C2-A new combined global gravity field model including GOCE data up to degree and order 1949 of GFZ Potsdam and GRGS Toulouse. EGU General Assembly Conference, Vienna

Helleputte TV, Doornbos E, Visser P (2009) CHAMP and GRACE accelerometer calibration by GPS-based orbit determination. Adv Space Res 43(12):1890-1896

Jäggi A, Beutler G, Mervart L (2010) GRACE gravity field determination using the Celestial mechanics approach-First results. Gravity, Geoid Earth Observ Int Assoc Geod Symp 135:177-184

Liu X (2008) Global gravity field recovery from satellite-to-satellite tracking data with the acceleration approach. Dissertation. University of Bonn, Bonn

Mayer-Gürr T (2006) Gravitationsfeldbestimmung aus der Analyse kurzer Bahnbögen am Beispiel der Satellitenmissionen CHAMP und GRACE, Dissertation. University of Bonn, Bonn

Mayer-Gürr T, Kurtenbach E, Eicker A, Kusche J (2010) ITG-Grace2010 gravity field model. www.igg. uni-bonn.de/apmg/index.Php

Prange L, Jäggi A, Beutler G (2011) AIUB-CHAMP03S: A gravity field model from eight years of CHAMP GPS data. www.aiub.unibe.ch/content/research/

Reigber C, Lühr H, Schwintzer P (2002) CHAMP mission status. Adv Space Res 30(2):129-134

Rieser D, Mayer-Gürr T, Savcenko R, Bosch W, Wünsch J, Dahle C, Flechtner F (2012) The ocean tide model EOT11a in spherical harmonics representation. URL: http://portal.tugraz.at/portal/page/portal/ Files/i5210/files/projekte/COTAGA/TN_EOT11a.pdf

Savcenko R, Bosch W (2012) EOT11a-empirical ocean tide model from multi-mission satellite altimetry. DGFI, Munich 89

Schall J, Eicker A, Kusche J (2014) The ITG-Goce02 gravity field model from GOCE orbit and gradiometer data based on the short arc approach. J Geod 88:403-409

Shen Y, Chen Q, Hsu H, Zhang X, Lou L (2013) A modified short arc approach for recovering gravity field model. Oral presentation at the GRACE Science team Meeting, Oct. 23-26, 2013, Center of Space Research, University of Texas

Tapley BD, Bettadpur S, Watkins M, Reigber C (2004) The gravity recovery and climate experiment: mission overview and early results. Geophys Res Lett 31:L09607

Tapley BD, Flechtner F, Bettadpur SV, Watkins MM (2013) The status and future prospect for GRACE after the first decade, Eos Trans Fall Meet Suppl., Abstract G22A-01

Zhao Q (2004) Research on precise orbit determination theory and software of both GPS navigation constellation and LEO satellites. PhD dissertation in Chinese with an abstract in English, School of Geodesy and Geomatics, Wuhan University, Wuhan

Zhao Q, Guo J, Hu Z, Shi C, Liu J, Cai H, Liu X (2011) GRACE gravity field modeling with an investigation on correlation between nuisance parameters and gravity field coefficients. Adv Space Res 47(10):1833-1850 